\title{
RECONSTRUCCIÓN DEL SISTEMA DE CONTROL DE UN EQUIPO DE LABORATORIO CLASIFICADOR DE PIEZAS POR PESO
}

\author{
Alfonso Valentín Poncela Méndez, Eduardo Julio Moya de la Torre, Fco. Javier García Ruiz \\ Instituto de las Tecnologías Avanzadas de la Producción, ITAP. \\ Escuela de Ingenierías Industriales, Paseo del Cauce 59, Valladolid, 47011, España. \{poncela, edumoy, \\ javgar\}@eii.uva.es \\ Mario Blázquez Gutiérrez \\ AVL Ibérica S.A. Departamento de Ingeniería eléctrica y automática. \\ Paseo Arco de Ladrillo 68, planta 5a, Valladolid, 47007, España. mario.blázquez@avl.com
}

\begin{abstract}
RESUMEN
El presente artículo, versa sobre la rehabilitación del sistema control de un equipo servocontrolado en posición, dotado de un manipulador electroneumático cuya función es la de clasificar y separar piezas en función de su peso. Este equipo está ubicado en el laboratorio de Automatización y Control del Departamento de Ingeniería de Sistemas y Automática de la Universidad de Valladolid. Fue puesto en servicio tiempo atrás usando medios de control y programación de la época, pero hoy en día fuera de uso. El equipo funciona correctamente, pero sólo vale como demostrador. Su función docente no es útil dada la antigüedad de los medios de control y programación empleados, de ahi la necesidad de su puesta al día.
\end{abstract}

Palabras clave: Autómatas programables, remodelación, programación, configuración, parametrización

\section{INTRODUCION}

En este trabajo se muestra la rehabilitación de un sistema mecánico, (adaptado a las nuevas tecnologías), electroneumático, servocontrolado que emplea autómata programable (PLC), control numérico de un eje (CNC) y dotado de interfaz hombre máquina (HMI). Su finalidad para la docencia es el ver en realidad el comportamiento de un sistema de producción clasificatorio de piezas para la obtención de datos que sirvan para la mejora de la organización de un sistema productivo, para ello se observa la recolección de piezas precedentes de una cadena de producción, clasificarlas en función de su peso y distribuirlas hacia el punto de utilización correspondiente.
Las piezas llegan paralelas al movimiento del eje servocontrolado y se pesan perpendiculares al movimiento, es decir hay que girarlas noventa grados antes de pesarlas. Todos los materiales empleados son de tipo industrial de marcas conocidas en entornos fabriles para familiarizar al estudiante con medios reales industriales.

El equipo ha sido diseñado, e implementado en el Departamento de Ingeniería de Sistemas y Automática (ISA) de la Escuela de Ingenierías Industriales (EII) de la Universidad de Valladolid (UVa) para desarrollar sus tareas docentes en distintas disciplinas, según sus necesidades.

En este caso se considera que es una práctica, (de tipo voluntario), imprescindible para los estudiantes del Grado de Ingeniería en Organización Industrial, estudiantes con pocos conocimientos en sistemas de automáticos de control para facilitar el uso del equipo y para ello, se pueden utilizar dos aplicaciones que corren en el PC asociado al sistema.

1) "programar" el ciclo de funcionamiento automático sin conocimientos sobre programación de PLC, y

2) "manejar" el sistema desde la pantalla en lugar de la botonera real anexa.

La amplia posibilidad de aplicaciones de uso del equipo, y la existencia de una única unidad, hace que los grupos de alumnos no puedan ser muy numerosos, lo que beneficia el aprendizaje del alumno, y aunque el coste inicial sea algo elevado, es fácilmente amortizable en pocos años.

El objetivo final, es ofrecer una formación básica y sólida para que el alumno adquiera las destrezas necesarias relacionadas con un sistema 
de producción industrial, tanto en su vida académica como profesional.

En definitiva, se pretende:

1. Motivar a los estudiantes impartiendo las clases prácticas de manera que aprecien los resultados de los problemas que están resolviendo.

2. Despertar en el alumno la curiosidad de ver lo que pueden llegar a hacer aplicando los contenidos estudiados.

Para lograr alcanzar estos objetivos se desarrolla esta práctica, en la que el alumno pueda plasmar los conocimientos adquiridos y vea los resultados por medio de distintos sistemas reales. Para ello se usará la plataforma TIA Portal de SIEMENS, tanto para configuración como la programación, que los alumnos han utilizado previamente en otras prácticas en el curso, basado en GRAFCET y contactos (LADDER, KOP).

Tras esta breve introducción, el artículo se estructura: en la segunda sección se realiza una breve descripción del sistema original, en la tercera sección se presenta la descripción de las cómo se ha procedido a la reconstrucción para adaptar la practica a los distintos grados y masters impartidos por el departamento. Por último se presentan las conclusiones obtenidas y las referencias utilizadas.

\section{SISTEMA ORIGINAL}

El sistema original, Figura 1, fue diseñado, montado y puesto en servicio de la mano de un proyecto fin de carrera [6], para la obtención del título de ingeniero industrial en la Escuela Técnica Superior de Ingenieros Industriales (hoy Escuela de Ingenierías Industriales EII) de la Universidad de Valladolid (UVa). Pese al tiempo transcurrido el sistema ha sido utilizado en tareas docentes, si bien apenas podían llevarse a cabo modificaciones, ni en la maniobra ni en la interface hombre máquina asociada, por el trabajo que ello entrañaba, y carecer de interés formativo.

El sistema era un mero demostrador de un automatismo industrial. Tanto el ordenador personal usado (PC 486 bajo W98) para la programación, como el sistema operativo, y la herramienta de programación STEP5 (bajo SO DOS), habían quedado completamente obsoletos.

En la Figura 2 se ve el cuadro de control original (no había armario, los medios estaban al aire sobre la placa de montaje naranja).

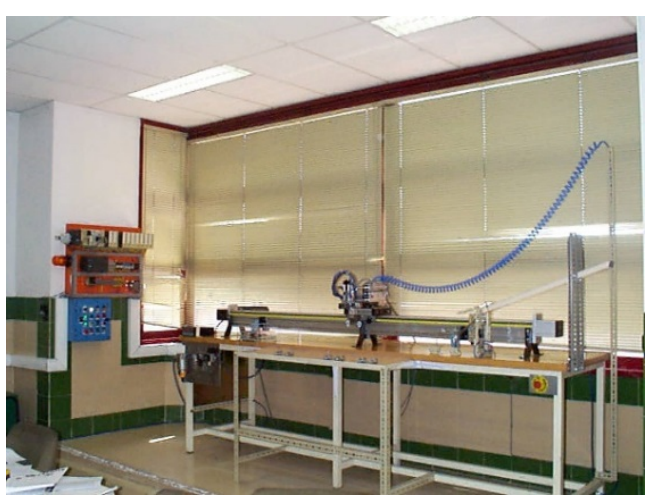

Figura 1: Sistema original

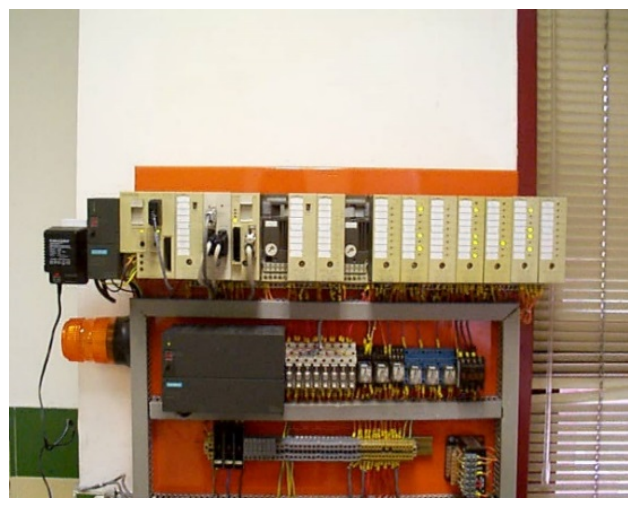

Figura 2: Detalle del PLC usado S5-CPU103

La Figura 3 muestra una de las pantallas del HMI desarrollado. La comunicación PLC-HMI PC se resolvió via comunicación serie RS232C programando en BASIC el procesador de comunicaciones usado.

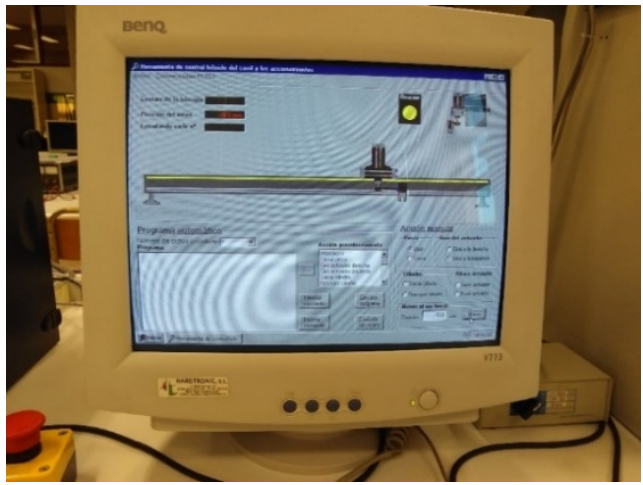

Figura 3: Interface hombre máquina desarrollado

La aplicación HMI hecha a medida con Visual Basic permitía no solo ver el estado del sistema sino, además, "programar" la maniobra automática sin necesidad trabajar en STEP5. Esta utilidad en el retrofitting propuesto se ha descartado, de modo que se deja el HMI únicamente para ver el estado del sistema y facilitar el diálogo hombre-máquina.

Una última comunicación relacionada con el sistema quedó reflejada en [5]. 
El trabajo, dado el volumen de tareas que implicaba (diseño del sistema de control, realización de esquemas eléctricos, fabricación, montaje, programación del PLC, programación del CNC de un eje IP266 -usado para controlar eje lineal-, identificación y modelado vía sistema de adquisición de datos Data Translation DT2801 -bus ISA-, usando Matlab y Simulink), fue adaptado a la nueva situación de las titulaciones actuales -EEES- cuyos equivalentes son los trabajos fin de grado y fin de master. La parte del retrofitting se asignó a un TFM [1], la parte HMI a un TFG [4], y se descartaron las restantes partes del trabajo original. El sistema queda abierto para ser usado en tareas docentes que impliquen programación de PLCs, programación de ejes lineales, comunicaciones e interface hombre máquina.

Las versiones originales de los proyectos creados en uno y otro caso una vez cargados en los sistemas, permiten evaluar su funcionamiento $\mathrm{y}$ mostrar a los estudiantes lo que ellos deben intentar resolver.

El modo de funcionamiento original era como sigue. Se disponía de modo manual y modo automático elegibles desde botonera física. En el modo manual el usuario via botonera desplazaba el manipulador libremente a ambos lados de la guía lineal (Bosch 2.5m) dentro de los límites físicos (cinco finales de carrera mecánicos uno para toma de referencia, dos finales ligados al control numérico y otros dos ligados a la fuente de alimentación del servomotor a modo de corte de seguridad). El movimiento se gestionaba con el servomotor 1FT5 de CA existente, dotado de encoder incremental 2500I/V, tacómetro, transmisión por polea con correa dentada y sin reductora alguna.

El tacómetro unido al módulo de avance y el encoder al CNC incluido en el PLC. Sobre la mesa de la guía estaba el manipulador neumático cuyos cilindros eran accionados desde el HMI únicamente (ocho acciones en total, cuatro electroválvulas cinco vías dos posiciones biestables, y sus correspondientes finales de carrera magnéticos), Figura 4.

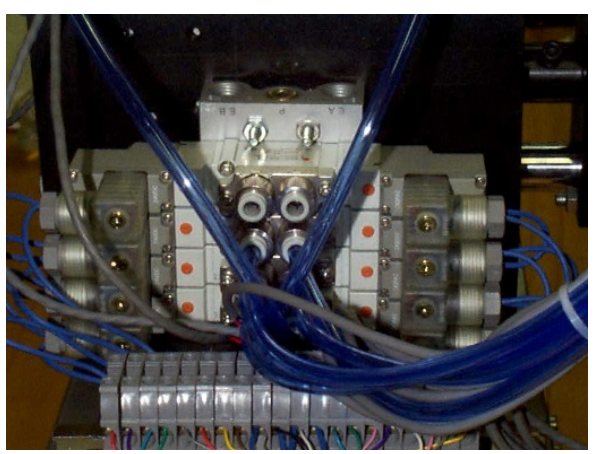

Figura 4: Placa electroválvulas del manipulador
La maniobra automática suponía: alimentar pieza (detección inductiva y electroválvula cinco vías retorno muelle), enviar al manipulador al puesto de alimentación, recoger pieza, llevar pieza a puesto de pesada, depositar pieza sobre la báscula, leer la pesada, retirar la pieza, desplazarla a la cota de salida correspondiente, evacuar pieza, alimentar pieza de nuevo y vuelta a empezar el ciclo hasta que no haya pieza nueva.

La zona de trabajo contaba con dos fotocélulas réflex (para impedir la entrada de personal en zonas con partes en movimiento), y tres setas de parada de emergencia con enclavamiento.

El ciclo automático no se optimiza (solapamiento de operaciones), por el eminente carácter didáctico que se pretende conferir al sistema.

\subsection{ELEMENTOS ADICIONALES}

Dado que el manipulador es completamente neumático, Figura 5, (cilindro con guías, cilindro rotolineal $0 / 90^{\circ}$ y pinza, todos ellos doble efecto) Figuras 6,7,8,9, así como el puesto de alimentación, el sistema incluye compresor alternativo cuyo aire es tratado (para no dañar las camisas de los cilindros), con una cadena de tratamiento de aire estándar: filtro antihumedad, filtro micropartículas, manorreductor, engrasador y electroválvula de arranque progresivo. No hay presostato, Figura 10.

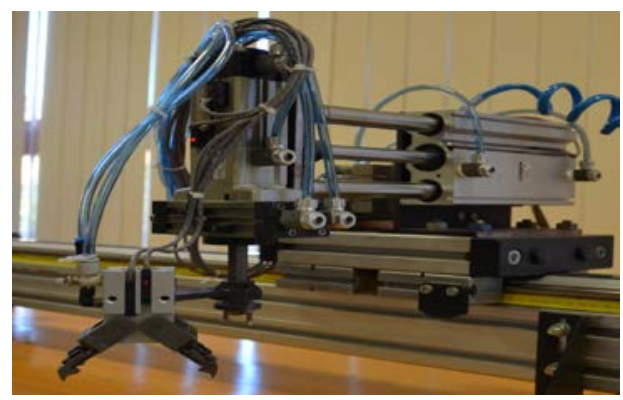

Figura 5: Manipulador
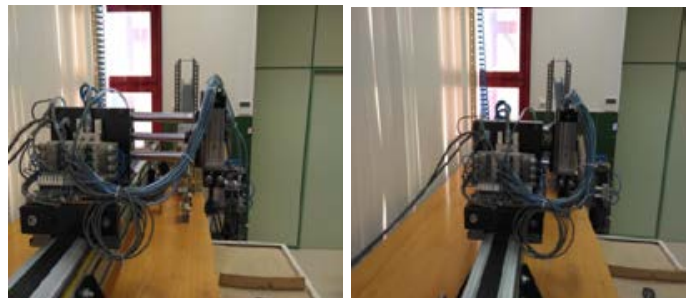

Figura 6: Cilindro guiado avance / retroceso

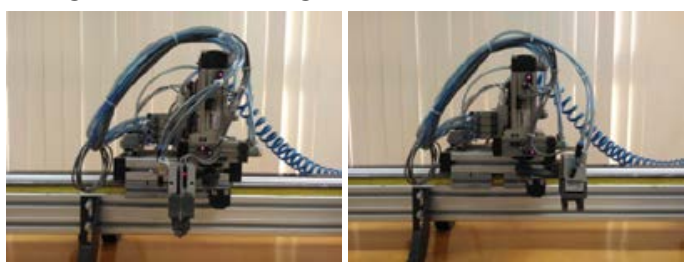

Figura 7: Cilindro rotolineal giro $0 / 90^{\circ}$ 


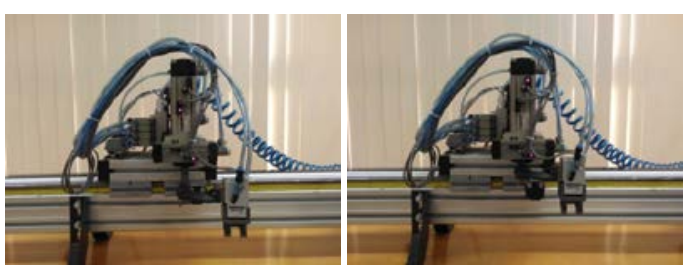

Figura 8: Cilindro rotolineal bajar / subir.

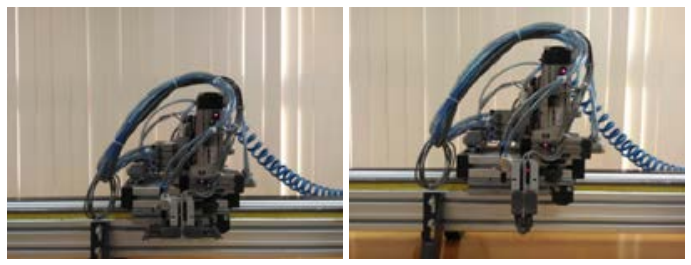

Figura 9: Pinza captura pieza (abierta, cerrada)

Para poder pesar, se colocó un plato de esquinas compensadas, dotado de una célula de carga de pesada máxima $7.2 \mathrm{Kg}$, y fue conectado a un acondicionador de señal Hottinger Baldwin Messtechnik (HBM), que proporciona una salida analógica $0-10 \mathrm{~V}$, con precisión de $\pm 2 \mathrm{~g}$. Para evitar posibles golpes, se protegió mediante un carenado de metacrilato y topes para limitar la carrera y proteger la célula de carga, Figura 11.

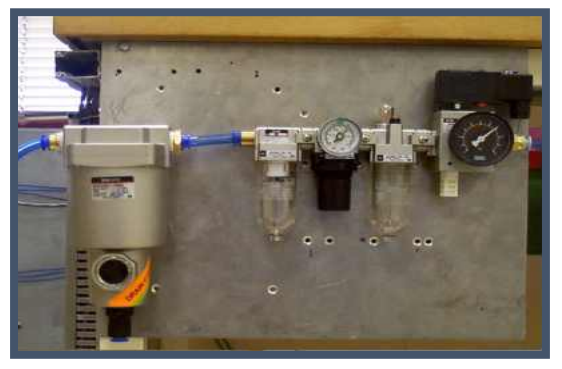

Figura 10: Unidad de tratamiento del aire
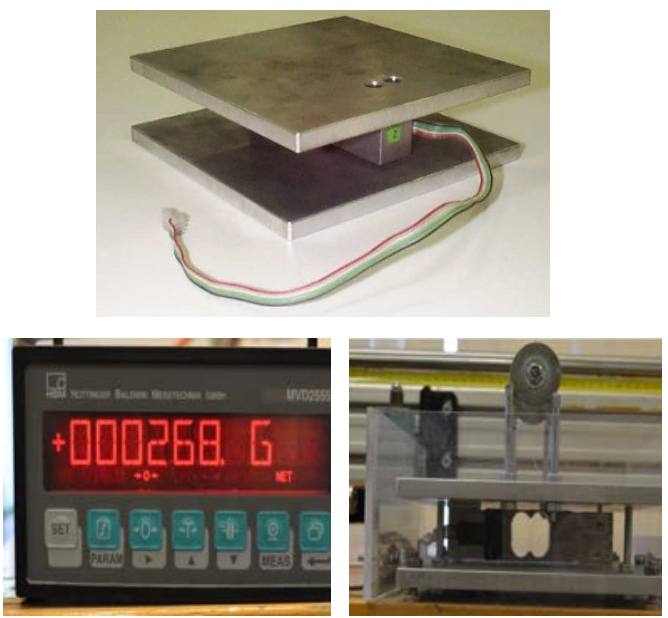

Figura 11: Acondicionador y célula de carga

\section{RECONSTRUCCIÓN}

La realización del trabajo supuso la ejecución de las siguientes tareas:

- Selección del equipo necesario.

- Planteamiento del hardware seleccionado sobre placa de montaje del armario.

- Realización de esquemas eléctricos con herramienta EPLAN Electric P8[2].

- Montaje del armario eléctrico (mecanizado placa de montaje, colocación de perfiles, colocación canaletas, anclaje de elementos, mecanizado del frontal, colocación de pulsantería, señalización, seccionador, cableado de todos los elementos dispuestos)

- Retirada de todos los elementos obsoletos (CPU S5-103, elementos de bus, procesador de comunicaciones CP521 SI, servomotor 1FT5, fuente de alimentación del servomotor $380 \mathrm{Vac} / 500 \mathrm{Vdc}$, módulo de avance $611 \mathrm{~A}$, CNC IP 262, fuente alimentación principal del PLC $24 \mathrm{Vcc}$ y fuente de alimentación auxiliar 24Vcc pulsantería y señalización), y su cableado.

- Mecanizado brida nueva para el nuevo servomotor y montaje sobre guía.

- Parametrización y puesta en marcha del variador S110 y módulo de control CU305.

- Programación de la maniobra del sistema.

- Revisión del cableado

- Puesta en marcha del sistema.

\subsection{Hardware seleccionado}

Para la reconstrucción del sistema se eligió el siguiente Hardware, todo ello de Siemens:

- Servomotor Simotics S-1FK7, Figura 12

- Variador Sinamics S110[11]: Power Module PM240-2 y Control Unit CU305. Figura 13

- CPU S7-1512C-1PN con $250 \mathrm{kB}$ memoria de trabajo. Por tratarse de una CPU compacta incluye: 32 entradas digitales; 32 salidas digitales; 5 entradas analógicas y 2 salidas analógicas. Adicionalmente como todas las CPUs se la serie S7-1500 cuenta con interfaz PROFINET integrado con switch de 2 puertos[10].

- Perfil soporte de $480 \mathrm{~mm}$.

- Fuente de alimentación PM1507, 24V/8A.

- SIMATIC Memory Card 24Mb.

- Fuente auxiliar para mando y señalización $24 \mathrm{Vcc} 10 \mathrm{~A}$.

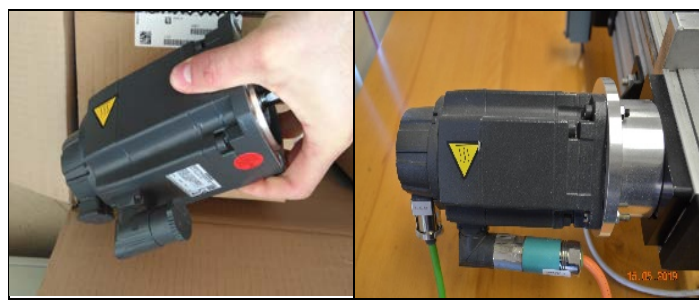

Figura 12: servomotor empleado

\subsection{Esquemas eléctricos}

Debido a la necesidad de recableado del nuevo armario fue ineludible la realización de unos nuevos esquemas actualizados acorde a este 
armario usando la herramienta anteriormente citada, EPLAN Electric P8[2].

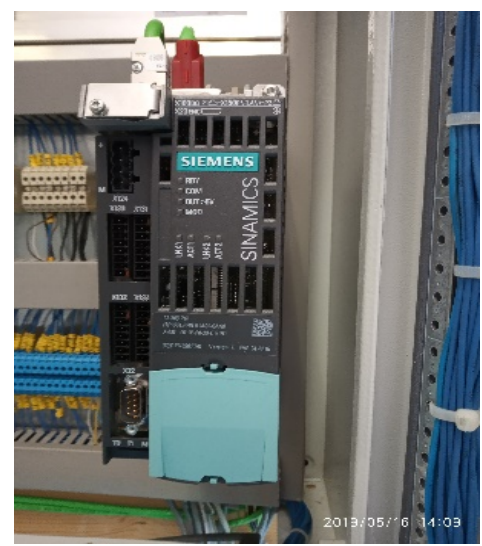

Figura 13: control del eje mesa

\subsection{Armario de control}

Para el presente proyecto se eligió una placa de montaje de $800 \mathrm{~mm}$ x $600 \mathrm{~mm}$ que se alojará en el armario de control, Figura 14.

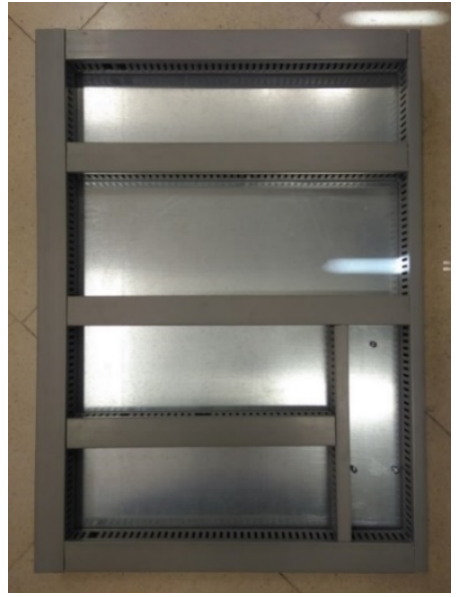

Figura 14: Placa de montaje con canaleta porta cables ya instalada

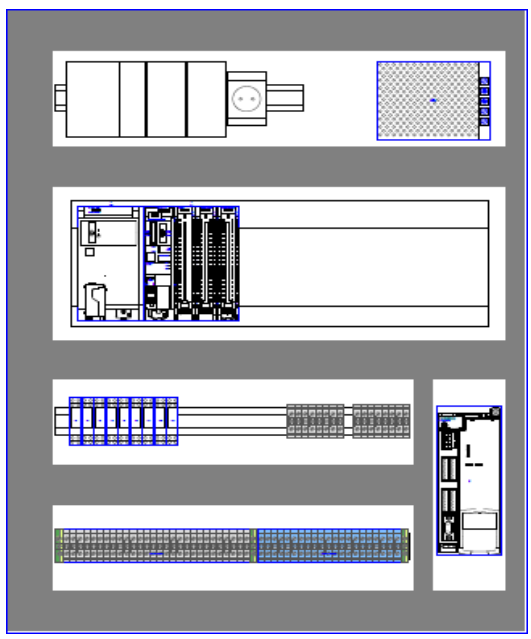

Figura 15: placa de montaje proyectada

Los elementos integrantes del nuevo sistema de control y los auxiliares necesarios (seccionador, diferencial magnetotérmicos, guardamotor, regleteros, relés auxiliares) se colocaron en sus posiciones y se cablearon conforme a los esquemas realizados, Figura 15.

Una vez fabricada la placa, Figura 16, se procedió al cableado de la puerta. Dado que el PLC elegido contaba con entradas y salidas suficientes, se decidió cablear pulsantería y señalización convencional suficiente para evitar la dependencia de un HMI adicional, y facilitar el uso del sistema en cursos que no impliquen diseño de interfaces hombre máquina, Figuras 17 y 18 .

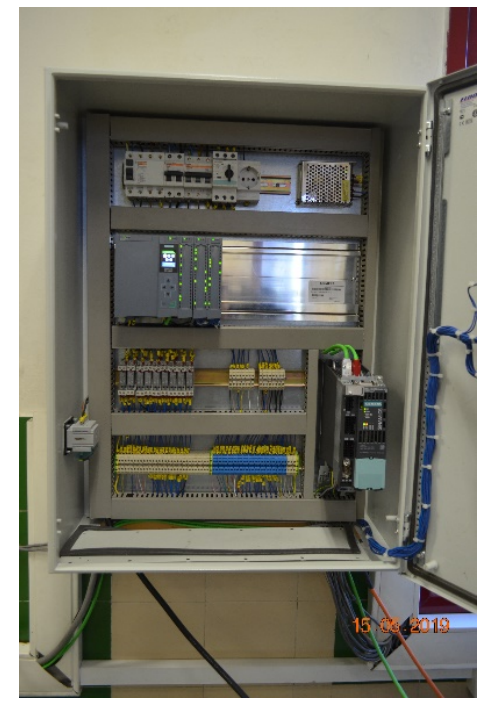

Figura 16: Vista del interior del nuevo armario de control ya colocado en posición

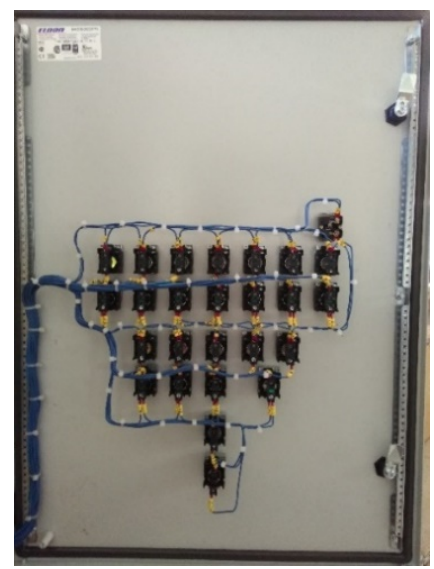

Figura 17: Vista de la parte interior de la puerta

\subsection{Configuración del sistema}

En la Figura 19, podemos ver la configuración de la red de comunicaciones elegida para la realización del presente trabajo. EL PLC y el controlador del eje se conectan a la red de comunicaciones del Departamento, de modo que 
desde cualquier laboratorio del mismo pueda accederse a todos ellos. El futuro HMI se podrá conectar el segundo puerto PROFINET del PLC o directamente a la red.

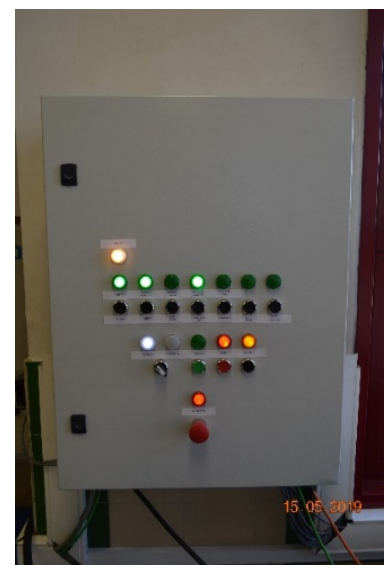

Figura 18: vista del frontal de la puerta

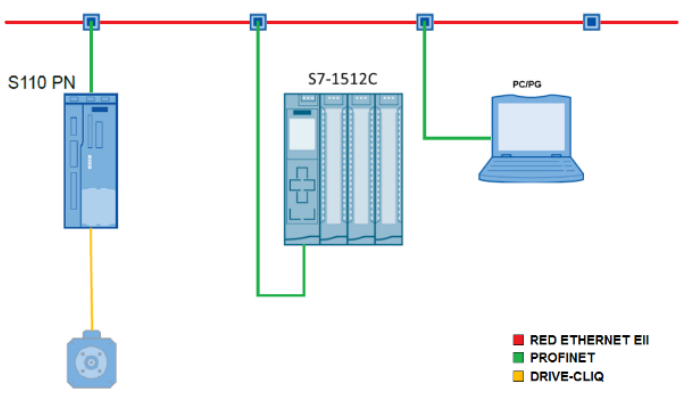

Figura 19: Mapa de comunicaciones elegido

\subsection{Configuración del variador}

Para la puesta en marcha del Sinamics S110, módulo de control CU305, se ha recurrido al programa "STARTER Engineering Tool".

Mediante esta herramienta se realiza la parametrización y puesta en servicio del variador S110[3],[9].

Ello supone:

$\checkmark$ Asignar dirección IP, definir la máscara de subred e indicar la dirección del router usado, para la gestionar las comunicaciones.

$\checkmark$ Restablecer la configuración de fábrica al variador.

$\checkmark$ Cargar el contenido del dispositivo en la consola de programación.

$\checkmark$ Definir el tipo de posicionamiento y el control de velocidad.

$\checkmark$ Asignar el módulo de potencia empleado.

$\checkmark$ Definir el motor empleado y complementos como retención mediante freno o DRIVECliq.

$\checkmark$ Precisar el tipo de encoder.

$\checkmark$ Asignar el sistema de medición y posicionamiento.
Definir las unidades de usuario que corresponden a un giro del eje.

$\checkmark$ Seleccionar el telegrama 111 de comunicación para el variador.

$\checkmark$ Asignar el tipo de comunicación marcando el tipo de telegrama (394).

$\checkmark$ Definir el control de la velocidad y se cargar el variador.

Para el ajuste del lazo de control fue necesario[8]:

$\checkmark$ Programar los límites máximos y mínimos para la aceleración.

$\checkmark$ Definir las rampas de aceleración y deceleración.

$\checkmark$ Indicar la velocidad de consigna.

$\checkmark$ Definir la ganancia.

$\checkmark$ Definir la acción integral.

$\checkmark$ Indicar el tipo de referenciado.

La comunicación entre el módulo $\mathrm{S} 110$ y la CPU S7-1512c[7], se lleva a cabo mediante puerto PROFINET de que disponen ambos elementos como ya se ha indicado anteriormente. Para enviar las instrucciones de funcionamiento desde el PLC al módulo S110, se ha desarrollado un protocolo de comunicación mediante el cual poder comandar el variador. Toda esta programación se ha guardado en una única FB para poder simplificar el programa del autómata.

\subsection{Programación del sistema}

La herramienta empleada en esta ocasión es TIA Portal de Siemens[12], versión profesional, apta para trabajar con controladores S7-1500 como es nuestro caso. La programación se ha llevado a cabo en lenguaje de contactos (KOP) para la mayoría de los bloques lógicos del programa, empleando Grafcet (GRAPH) para la programación de la secuencia automática.

La maniobra programada para el control de este sistema es la siguiente:

$\checkmark$ Para permitir la entrada de aire comprimido al sistema neumático el PLC debe estar en modo operativo RUN y además, es necesario pulsar el botón MARCHA del panel frontal del armario. Si esto sucede la salida asociada Q6.6 valdrá un 1 lógico. Si una vez activada esta salida se pulsa una seguridad o se pulsa el botón PARO del panel, la electroválvula de arranque progresivo que controla el paso del aire al sistema será desactivada. El estado de la salida asociada a la electroválvula cambiará pasará a ser un 0 lógico.

$\checkmark$ Para la habilitación del variador, el funcionamiento será el mismo que en el caso anterior. El variador se habilitará al pulsar el botón MARCHA del panel frontal y se 
detendrá al pulsar el botón PARO o ser activada alguna de las seguridades.

El sistema tendrá tiene modos de funcionamiento que se controlan mediante un selector de dos posiciones (MAN/AUTO). Sólo se tendrán en cuenta las órdenes de movimiento de los actuadores neumáticos cuando el selector MAN/AUTO de la botonera se encuentre en posición manual (MAN). Si por el contrario el selector se encuentra en posición automático (AUTO), el PLC debe ignorar cualquier acción que se le indique desde la botonera, exceptuando del botón de MARCHA, PARO, REARME y las seguridades (como pulsación de las setas de emergencia, células fotoeléctricas y finales de carrera de seguridad), que sí se tendrá en cuenta.

En el modo manual (MAN), cada botón del frontal del cuadro eléctrico está asociado a un cilindro neumático.

La Figura 20 resume la programación hecha.

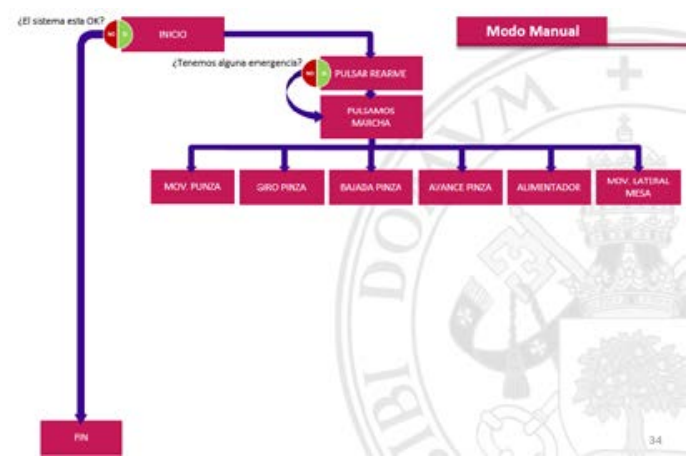

Figura 20: maniobra del PLC en modo manual

En el modo automático (AUTO), Figura 21, el sistema deberá hacer una tarea pre-programada en el autómata, que consiste en:

1. Búsqueda del cero de referencia en la guía.

2. Alimentar pieza sino hay pieza.

3. Avanzar hasta puesto de recogida de pieza.

4. Recoger pieza en el alimentador.

5. Abrir pinza, bajar pinza, recoger pieza, cerrar pinza y subir.

6. Ir a la báscula de pesaje.

7. Soltar pieza en la báscula.

8. Pesar pieza.

9. Recoger pieza de la báscula.

10. Clasificar según peso.

11. Ir al cajón correspondiente.

12. Soltar pieza en el cajón.

13. Volver a iniciar ciclo a partir del paso 2.

Esta secuencia comenzará al pulsar el botón MARCHA del panel frontal del armario y se detendrá al pulsar el botón PARO. Si realizamos dicha acción, el programa no perderá la información sobre la etapa del programa en la que se encuentra por lo que al volver a pulsar el botón MARCHA continuara el programa en el punto donde se quedó. Si por algún motivo una de las emergencias es activada el programa deberá volver a su inicio, por lo que para poder activar la secuencia se tiene que pulsar el botón REARME y seguidamente el botón MARCHA.

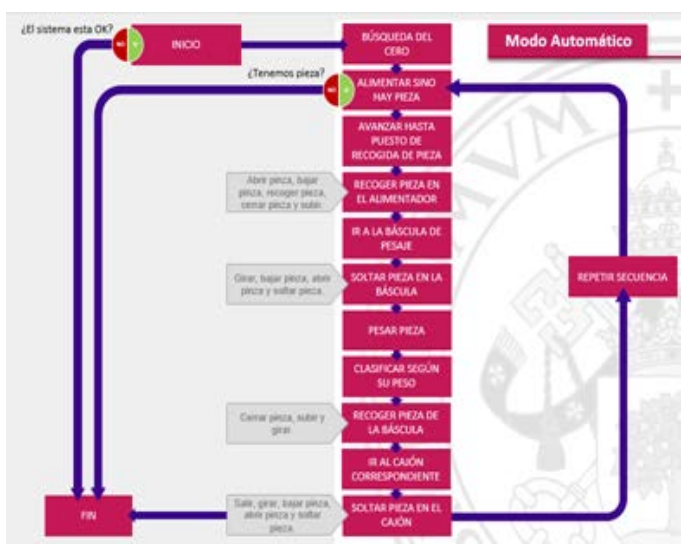

Figura 21: maniobra automática programada

La secuencia desarrollada al ser una secuencia realizada en Graph puede ser modificada o sustituida por otra que se adapte a posibles modificaciones del sistema. También puede ser sustituido por otra función permitiendo así la programación a los alumnos sirviéndoles de este modo como práctica de laboratorio.

Si se activan cualquiera de las emergencias, quedará enclavada hasta el momento en el que se pulse el botón del panel frontal de rearme. Si ésta señal permanece activa, no se permitirá el movimiento de ningún actuador ya que la entrada de aire al sistema queda bloqueada hasta el rearme.

\section{CONCLUSIONES}

Tras el trabajo realizado se extraen las siguientes conclusiones:

- Se ha realizado la rehabilitación y puesta en marcha del sistema de control para funcionamiento suave y seguro, con medios actuales.

- Se han realizado los esquemas eléctricos del armario y del conexionado de los elementos de campo.

- Se ha realizado el diseño, fabricación y montaje del nuevo armario eléctrico de control del sistema.

- Se ha programado y parametrizado el variador Simotics S110 consiguiendo el desplazamiento de la mesa del manipulador, suave, seguro, en ambas direcciones y a diferentes velocidades en función de la tarea a realizar.

- Se ha programado en el autómata S7-1512c una maniobra manual y otra automática que 
muestran a los alumnos el funcionamiento de un automatismo industrial montado en laboratorio.

El sistema queda a disposición del Departamento para programar las acciones formativas que con conjunto pueden desarrollarse: programación de PLCs de la serie 1500 de Siemens; manejo y parametrización del Control del Eje mediante el accionamiento Simotic y servomotor 1FK7; manejo de señales analógicas; manejo de sistemas con accionamientos neumáticos; realización de interfaces hombre máquina (HMI); comunicaciones industriales...

\section{Agradecimientos}

Los autores dan las gracias al Instituto de las Tecnologías Avanzadas de la Producción de la Universidad de Valladolid (ITAP UVa), al Departamento de Ingeniería de Sistemas y Automatica de la Universidad de Valladolid (ISA UVa) por los medios económicos y recursos materiales facilitados para la realización de este proyecto, y a la Delegación de Siemens en Valladolid por su apoyo material y técnico.

\section{SYSTEM CONTROL RETROFITTING OF A SYSTEM LAB CLASSIFIER BY WEIGHT}

\begin{abstract}
This paper describes the control system retrofitting of a servo controlled equipment that moves a pneumatic manipulator in classifying pieces on the basis of their weight. The equipment is located in the Control laboratory of the Department of Systems Engineering and Control. It was started up long time ago using hardware and software of the time. Nowadays, the system works properly, but it is only useful as demonstrator. It has little educational value, hence the need of updating.
\end{abstract}

Keywords: Programmable Logic Controller, programming, configuration, parametrization.

\section{Referencias}

[1] Blázquez, M., 2017 "Retrofitting de un sistema manipulador electro-neumático servoncontrolado clasificador de piezas por peso mediante PLC S7-1500", Trabajo de Fin de Máster, Universidad de Valladolid.
[2] Eplan Electric $\quad$ P8 https://www.eplan.es/es/soluciones/ingen ieria-electrica/eplan-electric-p8/ (último acceso mayo 2019)

[3] Honcik, Tomas, 2012 "Sinamics S110, puesta en marcha", Nürnberg, Alemania,

[4] Martín Martín, S. 2018, "Retrofitting del Sistema de Monitorización de un Manipulador Electroneumático Clasificador de Piezas por Peso Servocontrolado con HW SIEMENS S71500". Trabajo Fin de Grado, Universidad de Valladolid.

[5] Moya, E., Poncela, A.V., 2011, Equipo de Prácticas para Laboratorio de Automatización y Control con Material Industrial. Seminario Anual de Automática, Electrónica Industrial e Instrumentación SAAEI 2011 Actas del congreso XVIII edición SAAEI ISBN 978-84-933682-3-4 5-8 julio, 2011 Badajoz España págs. 657-662.

[6] Sáez, J., 1999, "Manipulador electroneumático controlado en posición mediante servomotor de C.A.", Valladolid, Universidad de Valladolid.

[7] Siemens, 2011 "Comunicación Sinamics S110 con el sistema de control Simatic S7-1200 a través de PROFINET", Siemens, Nüremberg, Alemania, 2011.

[8] Siemens, 2012 "Servo Drive Optimization Guide", Siemens, Nüremberg, Alemania, 2012.

[9] Siemens, 2013 "FAQ for changing and saving parameters using STARTER", Siemens, Nürenberg, Alemania 2013.

[10] Siemens, 2014 "Simatic S7-1500, manual". Nüremberg, Alemania.

[11] Siemens, 2015 "Sinamics S110, manual" Siemens Support, Siemens, Nüremberg, Alemania 2015.

\section{[12] TIAPortal}

https://w5.siemens.com/spain/web/es/ind ustry/automatizacion/simatic/tiaportal/pages/tiaportal.aspx, (último acceso mayo 2019) 